\section{Assessment of three-dimensional biofilm structure using an optical microscope}

\author{
Carla C.C.R. de Carvalho and M. Manuela R. da Fonseca \\ Instituto Superior Técnico, Lisbon, Portugal
}

BioTechniques 42:616-620 (May 2007)

doi 10.2144/000112403

A method allowing the evaluation of the structure and the calculation of the volume of a biofilm, using an optical microscope, is proposed based on the linear relation between the intensity of a pixel in biofilm images grabbed on the $x$-y plane and the corresponding number of cells in the $z$ direction, which allows the calculation of the biofilm thickness. The method is intended to overcome the need for expensive microscopes to study biofilms.

\section{INTRODUCTION}

Analysis of biofilm structure requires the acquisition of threedimensional (3-D) data. Transmission electron microscopy (TEM) and scanning electron microscopy (SEM) have been used to assess the structure of biofilms $(1,2)$. Although these techniques provide high-resolution images, they have limitations in sample penetration and require dehydration of the sample, which may result in disruptive shrinkage and loss of biofilm matrix $(3,4)$. Fluorescence in situ hybridization (FISH) presents the latter disadvantage, but it is a good technique to identify microorganisms in biofilms due to the use of specific fluorescently labeled nucleic acid probes $(5,6)$. To achieve this, FISH requires multicolor fluorescence microscopy, which is usually performed by confocal laserscanning microscopy (CLSM). CLSM is the most often used technique to acquire 3-D fluorescence microscopy data, since it permits in situ nondestructive study of hydrated living biofilms (7-9). It also allows the study of the physical structure of biofilms, the monitoring of biofilm development, and the assessment of biofilm response to the environment (10). The images obtained from CLSM are almost free of out-of-focus sections, because only a small fraction of the out-of-focus fluorescence emission can pass through the pinhole aperture. However, this also prevents the acquisition of a large part of the fluorescence emitted by the sample, reducing detection sensitivity and requiring illumination of the sample with high light intensity that may cause bleaching of fluorescent dyes. Furthermore, confocal laserscanning microscopes able to detect different fluorochromes can be expensive and are not available in many research centers.

The aim of the present work is to find a way of assessing the 3-D structure of a biofilm using an optical microscope with bright-field transmitted and fluorescent light. With a common optical microscope, it is almost impossible to capture stacks of images at defined distances to be used in the construction of 3-D images needed to characterize a biofilm. However, when a biofilm is observed using an optical microscope under bright-field transmitted light, it is possible to observe that the presence of cells in the $x-y$ plane decreases proportionally the amount of light that passes the sample in the perpendicular z-axis. The same principle is used in optical density determinations in spectrophotometers to calculate the amount of biomass in samples. To be able to determine the biofilm thickness, a relation between the amount of light observed in the two-dimensional (2-D) images and the number of cells in the vertical plane was needed. To determine the number of cells stacked in the z-axis responsible for a given pixel intensity in the $\mathrm{x}$ $y$ image, the biofilms were frozen with liquid nitrogen, and the stained cells were observed under fluorescent light.

The method was validated and applied in the study of the influence of solvents on biofilm formation in the wake of our studies on solvent toxicity in organic-aqueous systems (11).
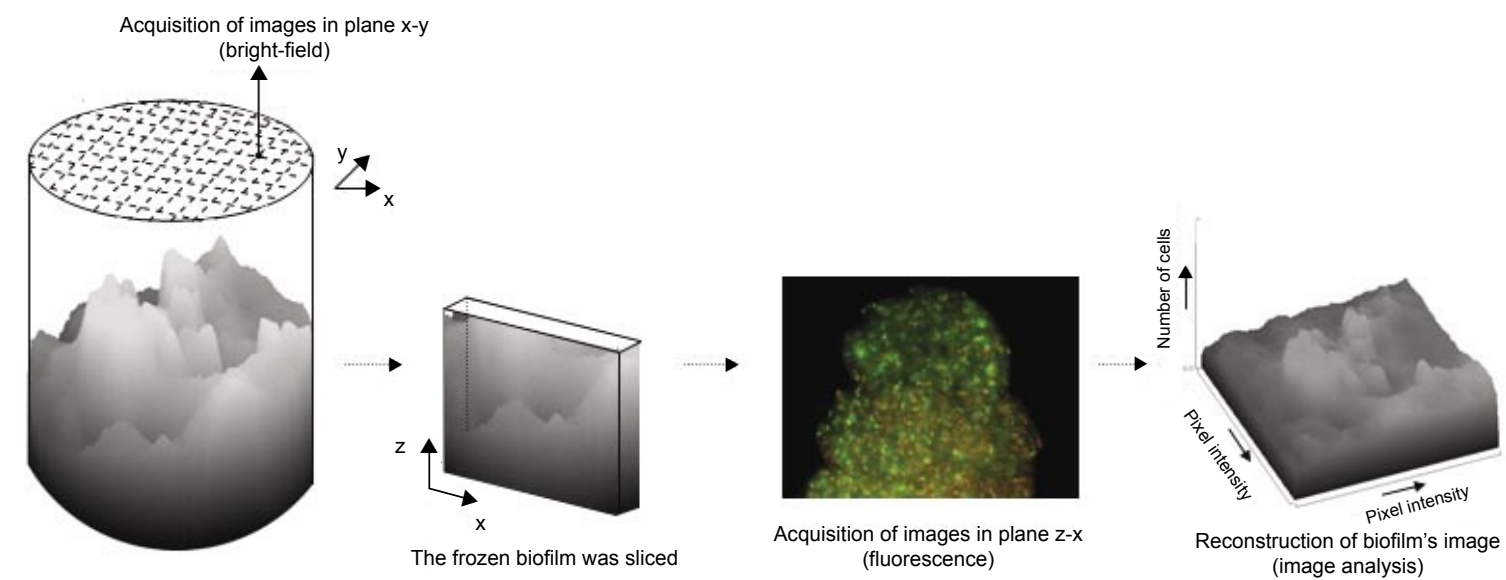

Figure 1. Representation of the method proposed to construct three-dimensional (3-D) images from two-dimensional (2-D) images of biofilms. 


\section{Short Technical Reports}

\section{MATERIALS AND METHODS}

\section{Biofilm Formation}

Rhodococcus erythropolis DCL14 cells were grown on 6-well plates (BD Falcon ${ }^{\mathrm{TM}}$ polystyrene tissue-culture plate; BD Biosciences, San Jose, CA, USA). Two hundred fifty microliters solvent were added to the $5-\mathrm{mL}$ cell suspension. The solvents tested were the following: $99.8 \%$ ethanol and $>99.5 \%$ toluene from Merck KGaA (Darmstadt, Germany), 99\% pentane from Fluka Chemie GmbH (Seelze, Germany), $>99 \% n$-hexane and $>99.5 \%$ iso-octane from Honeywell Riedelde Haën (Seelze, Germany), 95\% $n$-heptane from Lab-Scan (Hasselt, Belgium), >99\% n-octane from MerckSchuchardt (Hohenbrunn, Germany), 99\% n-nonane from Acros (Geel, Belgium), and $>99 \% n$-dodecane and 99\% $n$-hexadecane from Sigma-Aldrich Chemie GmbH (Buchs, Switzerland). The assays were done in triplicate. The biofilms formed were observed after 48 h. Cell hydrophobicity was measured as described by de Carvalho and da Fonseca (12).

\section{Biofilm Observation}

Method calibration. Each well of the 6-well plates containing the biofilms was observed under brightfield transmitted light with a $20 \times$ lens (N.A., 0.4), and images were taken on the intersection point of a grid with lines $1 \mathrm{~mm}$ apart. After staining the

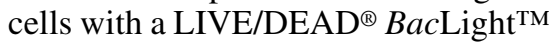
Bacterial Viability kit (Molecular Probes $^{\circledR}$; Invitrogen, Carlsbad, CA, USA), the content of the well was frozen with liquid nitrogen and sliced into 1-mm-thick slabs. Each slice, placed on a glass slide, was observed over a glass Petri dish (containing ice to keep the samples frozen) under fluorescent light with a $100 \times$ lens (N.A., 1.3). Images showing the number of stacked cells were taken 1 $\mathrm{mm}$ apart from each other. The microscope used was an Olympus CX40 with an Olympus U-RFL-T burner and an U-MWB mirror cube unit (excitation filter, BP450-480; barrier filter, BA515; Olympus, Center Valley, PA, USA). Images were captured by a
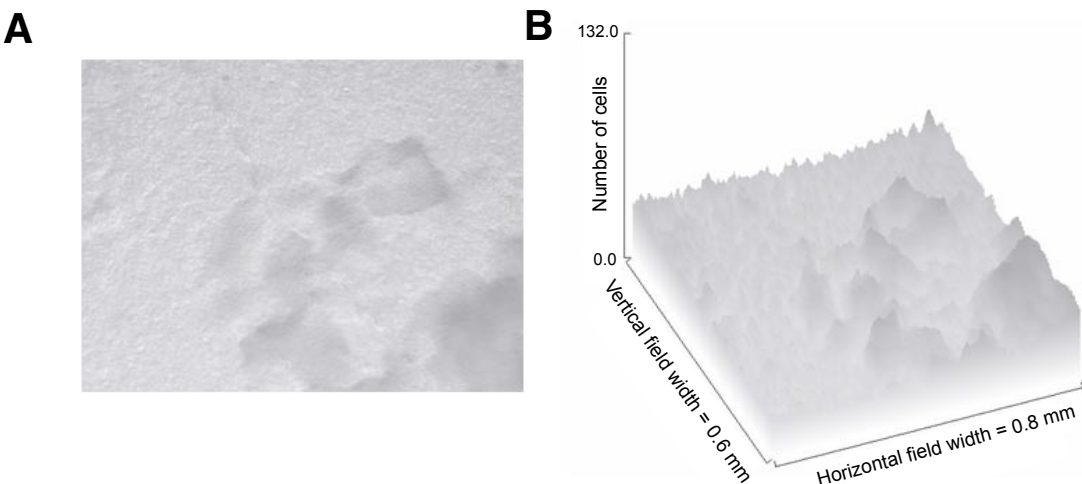

Figure 2. Biofilm images. (A) After conversion into grayscale (horizontal field width, $0.4 \mathrm{~mm}$; vertical field width, $0.3 \mathrm{~mm}$; N.A., $20 \times$ lens, 0.4). (B) Corresponding surface plot obtained using ImageJ representing the number of cells in panel A.

Cohu RGB camera (Cohu, San Diego, CA, USA). The acquisition software was Matrox Inspector 2.1 (Matrox Imaging, Dorval, QC, Canada).

Biofilm studies. Each well of the 6-well plates containing the biofilms grown in the presence of the solvents to be tested was observed under brightfield transmitted light with a $20 \times$ lens (N.A., 0.4), and the images were taken on the intersection point of a grid with lines $1 \mathrm{~mm}$ apart. Only $75 \%$ of the area of the well was analyzed-a circular corona 2-mm-wide on the edge of the wells was discarded to avoid interferences by fluid dynamics and by light reflections from the plastic of the multiwell plates. Using the calibration described in the section entitled Image Analysis, a 3-D image of the biofilm was obtained using ImageJ for each interception point.

\section{Image Analysis}

The images obtained under brightfield transmitted light in the RGB system were converted into grayscale images, and the intensity of each pixel was determined using ImageJ (rsb. info.nih.gov/ij; this software and its Java source code are freely available and no license is required for its use). Data containing arrays of the type $(x, y$, intensity) where $\mathrm{x}$ and $\mathrm{y}$ are the coordinates of the pixel positioning were collected using ImageJ. The images with cells visualized in the $\mathrm{z}$ direction under fluorescent light were analyzed using Visilog 5 (Noesis, Les Ulis, France) according to Reference 13 . The number and morphological parameters of both viable and nonviable cells were collected. The size of objects in images was calibrated using a TetraSpeck ${ }^{\mathrm{TM}}$ Fluorescent Microspheres Sampler kit (Molecular Probes; Invitrogen). A linear relation between the intensity value and the number of cells at each positioning was determined with $R^{2}$ $=0.957$. The equation describing the relation is:

$N C=(-0.664 \pm 0.056) \times I+(132.44 \pm 7.96)$

where $N C$ is the number of cells, and $I$ is the intensity.

Thirty images from 10 different biofilms (300 images total) were used to obtain the equation. This relation is valid for intensity values between 18 and 195, which corresponds to a minimum of 3 cells and a maximum of 120 cells. Below that range, the light is too bright and no differences in intensity are visible. On the other hand, if more than $120 \mathrm{R}$. erythropolis cells are piled, the field becomes too dark, and intensity values are not distinguishable. The linear correlation is valid for growing and fully grown biofilms as long as differences in light intensity may be observed throughout the biofilm. For thicker biofilms, more powerful light sources may be used as long as no damage is done to the cells or to the biofilm integrity.

Similar calibrations should be performed if this method is to be used, as the relation parameters could be influenced by the strain (some strains may be more opaque than others), the production of exopolymeric substances, and even technical conditions (e.g., microscope and camera used). The 
number of images necessary to obtain a good calibration equation depends on the amount of information that is given by a single image. If one image is too homogeneous, more images are necessary to quantify the number of cells in the whole array of possible cell stacks.

In the present case, the horizontal field width of the images had 768 pixels corresponding to $0.4 \mathrm{~mm}$, and the vertical field width had 576 pixels corresponding to $0.3 \mathrm{~mm}$. This and the linear regression relating the number of cells and intensity allowed the calibration of ImageJ and the compilation of a text file containing arrays (x, y, number of cells) in $(\mathrm{mm}, \mathrm{mm}$, number of cells, respectively) for each image. A surface plot could thus be obtained for each image obtained under bright-field transmitted light, allowing the assessment of 3-D data from 2-D images of biofilms.

\section{Surface and Volume Calculations}

The surface and volume calculations were performed using the Surfer ${ }^{\circledR}$ package from Golden Software (Golden, CO, USA). The calibration of the size of objects in images allowed the calculation of the average projected area and volume of a cell, which was considered as an ellipsoid. R. erythropolis cells presented an average elongation factor of 1.47 and a radius of 9.7 and $6.6 \mu \mathrm{m}$ in the longest and shortest axes, respectively. The volume of the biofilm was calculated by integration according to the trapezoidal rule (14). The surface of the biofilms refers to the total area in the $x-y-z$ dimension. The surface and volume data of each image captured were used to calculate the biofilm volume of the total surface analyzed. Although thousands of images were used to calibrate and validate the method, statistical analysis showed that approximately 50 images/well are sufficient to infer on the volume and area of the biofilm (data not shown).

\section{RESULTS AND DISCUSSION}

Figure 1 shows a representation of the proposed method to determine the volume of a biofilm from 2-D images. To validate the applicability of the method, the effect of solvents on biofilm formation was studied. Images of the biofilms grown in the presence of the different solvents were obtained

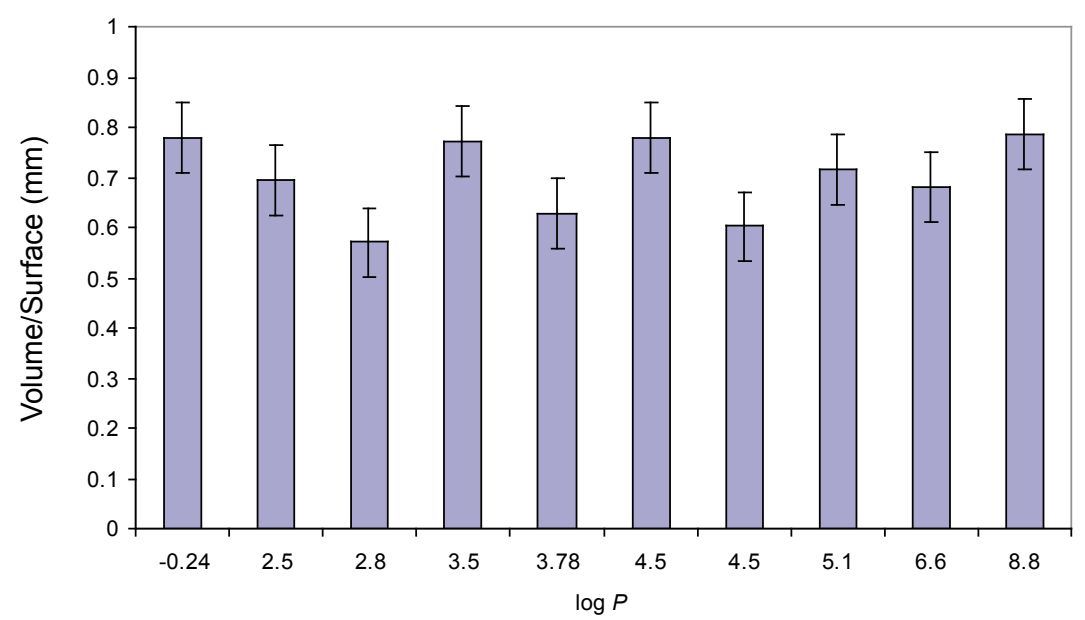

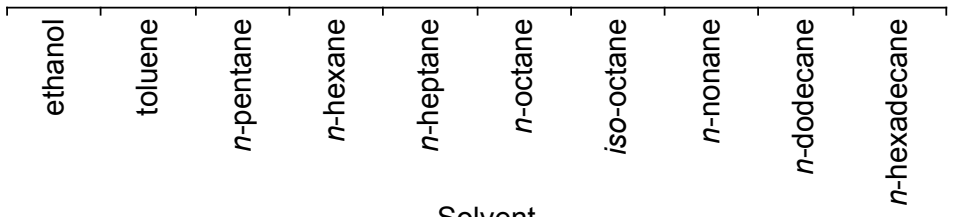

Solvent

Figure 3. Volume/surface ratio of the biofilm formed in the presence of solvents with different log $P$ values.

under bright-field transmitted light in the RGB system and converted into grayscale images (Figure 2A). The calibration equation was then used to obtain the number of cells corresponding to each pixel, which allowed the calculation of the volume of biofilm shown in each picture (Figure 2B)

According to Stoodley et al. (15), the biofilm structure may be influenced by the surface where the biofilm attaches, the properties of the microorganism in the biofilm, the hydrodynamic conditions, and the nutrient availability. In the present study, the surface where the cells attached, the microorganism, and the hydrodynamic conditions were kept constant in the various assays. The effect of the presence of the organic solvents, used as single carbon and energy source, was thus the only parameter affecting biofilm formation.

The $\log P$ value of a solvent, which measures the partition of the solvent in an octanol-water system, has been used to assess its toxicity (16): solvents with $\log P$ values higher than 4 are generally biocompatible, while those with values lower than 2 are not. Those with $\log P$ values between 2 and 4 have an unpredictable biocompatibility.

The volume/surface area ratios of the biofilms formed in the presence of solvents with $\log P$ values ranging from -0.24 (ethanol) to 8.8 ( $n$-hexadecane) was, on average, $0.70 \mathrm{~mm}$ (Figure 3), which indicates that the biofilms formed presented a rather smooth surface. Biofilms produced in the presence of $n$-pentane, $n$-heptane, and iso-octane presented a volume/surface ratio lower than the average value, while higher values were observed when the biofilms were formed in the presence of ethanol, $n$-hexane, $n$-octane, and $n$-hexadecane. Cells in biofilms with a low volume/ surface value have an easier access to nutrients than those in biofilms with a high volume/surface ratio. The latter, however, provides more protection to the cells, as the surface available for direct cell-medium contact is smaller. $R$. erythropolis DCL14 cells are able to grow on alcohols and $n$-alkanes (12). The growth rate is high when the cells grow on $0.125 \%$ and $0.25 \%(\mathrm{v} / \mathrm{v})$ ethanol. On even-numbered $n$-alkanes at the same concentrations, the growth rate increases with increasing number 
of carbon atoms of the carbon source. However, the growth rate decreases with increasing solvent concentration and is always very slow in the presence of odd numbered $n$-alkanes, apparently due to a less efficient mechanism for producing propionyl-CoA, the precursor of fatty acids containing an odd-numbered chain of carbon atoms. A relation between the chain length of the growth substrate and the chain length of the produced fatty acids was found with the strain $R$. erythropolis 17 (17). In the present work, a rough variant of the strain $R$. erythropolis DCL14 was chosen (i.e., the strain did not produce extracellular polymeric substances). Modifications of the cell surface hydrophobicity through alterations in the lipid composition of the membrane were probably the main mechanism responsible for cell adhesion in the presence of the different solvents tested.

Prior to the formation of a biofilm, in the presence of water immiscible solvents, $R$. erythropolis cells are mainly located at the organic-aqueous interface or even in the organic phase due to their high hydrophobicity. In contrast, in the presence of miscible ethanol, the cells are freely suspended in the aqueous phase (3). The dispersion of immiscible solvent droplets increases the number of possible points of cell adhesion, and a nonhomogeneous rough biofilm could be expected. However, the results obtained for the volume/surface ratio indicate the opposite. Cell hydrophobicity seems to be determinant to the volume of biofilm formed. Cells with a lower hydrophobic character were obtained in the presence of ethanol and alkanes with an odd number of carbon atoms (data not shown). In the presence of these compounds, cells apparently need more protection and aggregate. The resulting biofilms have, in average, larger volumes than those in the presence of less toxic alkanes, to which hydrophobic cells adhere easily.

In summary, the method described here allows the study of the structure of biofilms using an optical microscope and can be a useful tool in research centers that do not possess a confocal laser-scanning microscope.

\section{ACKNOWLEDGMENTS}

This work was supported by a postdoctoral grant (SFRH/BPD/14426) 2003) awarded to C.C.C.R.de.C. by Fundação para a Ciência $e$ a Tecnologia, Portugal.

\section{COMPETING INTERESTS STATEMENT}

The authors declare no competing interests.

\section{REFERENCES}

1. Kämper, M., S. Vetterkind, R. Berker, and M. Hoppert. 2004. Methods for in situ detection and characterization of extracellular polymers in biofilms by electron microscopy. J. Microbiol. Methods 57:55-64.

2. Singleton, S., R. Treloar, P. Warren, G.K. Watson, R. Hodgson, and C. Allison. 1997. Methods for microscopic characterization of oral biofilms: analysis of colonization, microstructure, and molecular transport phenomena. Adv. Dent. Res. 11:133-149.

3. Handley, P.S., N.A. Sutton, and N. Hughes. 1993. Problems associated with electron microscopy of biofilms, p. 61-66. In J. Wimpenny, W. Nichols, D. Stickler, and H. Lappin-Scott (Eds.), Bacterial Biofilms and Their Control in Medicine and Industry. Bioline, Cardiff, UK.

4. Lawrence, J.R., G.D.W. Swerhone, G.G. Leppard, T. Araki, X. Zhang, M.M. West, and A.P. Hitchcock. 2003. Scanning transmission $\mathrm{x}$-ray, laser scanning, and transmission electron microscopy mapping of the exopolymeric matrix of microbial biofilms. Appl. Environ. Microbiol. 69:5543-5554.

5. Hörsch, P., A. Gorenflo, C. Fuder, A. Deleage, and F.H. Frimmel. 2005. Biofouling of ultra- and nanofiltration membranes for drinking water treatment characterized by fluorescence in situ hybridization (FISH). Desalination 172:41-52.

6. Manz, W., U. Szewzyk, P. Ericsson, R. Amann, K.H. Schleifer, and T.-A. Stenström. 1993. In situ identification of bacteria in drinking water and adjoining biofilms by hybridization with $16 \mathrm{~S}$ and $23 \mathrm{~S}$ rRNA-directed fluorescent oligonucleotides probes. Appl. Environ. Microbiol. 59:2293-2298.

7.Caldwell, D.E., D.R. Korber, and J.R. Lawrence. 1992. Confocal laser microscopy and digital image analysis in microbial ecology. Adv. Microb. Ecol. 12:1-67.

8. Gu, F., R. Lux, L. Du-Thumm, I. Stokes, J. Kreth, M.H. Anderson, D.T. Wong, L. Wolinsky, et al. 2005. In situ and non-invasive detection of specific bacterial species in oral biofilms using fluorescently labeled monoclonal antibodies. J. Microbiol. Methods 62:145-160.

9. Yun, M.-A., K.-M. Yeon, J.-S. Park, C.H. Lee, J. Chun, and D.J. Lim. 2006 Characterization of biofilm structure and its effect on membrane permeability in MBR for dye wastewater treatment. Water Res. 40:4552

10. Beyenal, H., C. Donovan, Z. Lewandowski, and G. Harkin. 2004. Three-dimensional biofilm structure quantification. J. Microbiol. Methods 59:395-413.

11. de Carvalho, C.C.C.R. and M.M.R. da Fonseca. 2004. Solvent toxicity in organicaqueous systems analysed by multivariate analysis. Bioprocess Biosyst. Eng. 26:361375.

12. de Carvalho, C.C.C.R. and M.M.R. da Fonseca. 2005. Degradation of hydrocarbons and alcohols at different temperatures and salinities by Rhodococcus erythropolis DCL14. FEMS Microbiol. Ecol. 51:389-399.

13. de Carvalho, C.C.C.R., M.-N. Pons, and M.M.R. da Fonseca. 2003. Principal components analysis as a tool to summarise biotransformation data: influence on cells of solvent type and phase ratio. Biocatal Biotransformation 21:305-314.

14. Press, W.H., B.P. Flannery, S.A. Teukolsky, and W.T. Vetterling. 1988. Numerical Recipes in C. Cambridge University Press, Cambridge, UK.

15. Stoodley, P., J.D. Boyle, I. Dodds, and H.M. Lappin-Scott. 1997. Consensus model of biofilm structure, p. 1-9. In J.W.T. Wimpenny, P. Handley, P. Gilbert, H. Lappin-Scott, and M. Jones (Eds.), Biofilms: Community Interactions and Control. Bioline, Cardiff, UK.

16. Laane, C., S. Boeren, K. Vos, and C. Veeger. 1987. Rules for optimization of biocatalysis in organic solvents. Biotechnol. Bioeng. 30:81-87.

17. Alvarez, H.M. 2003. Relationship between b-oxidation and the hydrocarbon-degrading profile and actinomycetes bacteria. Int Biodeterior. Biodegradation 52:35-42.

Received 30 May 2006; accepted 22 December 2006.

Address correspondence to Carla C.C.R. de Carvalho, IBB-Institute for Biotechnology and Bioengineering, Centre for Biological and Chemical Engineering, Instituto Superior Técnico, Av. Rovisco Pais, 1049-001 Lisboa, Portugal. e-mail: ccarvalho@ist.utl.pt

To purchase reprints of this article, contact: Reprints@BioTechniques.com 